\title{
A proton imagining via double parton scattering
}

\section{Rinaldi*}

Dipartimento di Fisica e Geologia. Università degli studi di Perugia. INFN section of Perugia, via A. Pascoli, Perugia, Italy.

E-mail: matteo.rinaldi@pg.infn.it

In this contribution we discuss the main outcomes of our studies on the so called double parton distribution functions (dPDFs), accessible quantities in high energy proton-proton and proton nucleus collisions, in double parton scattering processes (DPS). These new distributions are almost unknown, nevertheless they encode information on how partons inside a proton are correlated among each other. Double PDFs represent a new tool to explore the three dimensional partonic structure of hadrons. Here, we show results obtained from calculations of dPFDs. In particular we focus our attention on the impact of double correlations in experimental observables by showing how the latter could be studied in the next LHC run. We also discuss how the present knowledge on a peculiar experimental observable could unveil new information on the transverse proton structure.

Light Cone 2019 - QCD on the light cone: from hadrons to heavy ions - LC2019

16-20 September 2019

Ecole Polytechnique, Palaiseau, France

${ }^{*}$ Speaker. 


\section{Introduction}

A proper description of multiple parton interactions (MPI) in hadron-hadron collisions, occurring when more than one parton of a hadron interact with partons of the other colliding hadron, is relevant thanks to the high luminosity reached in collider experiments, such as at the LHC. In addition MPI represent a background for the search of new Physic at the LHC. Since, MPI contribution to the total cross section is suppressed with respect to the single partonic interaction, the measurement of their cross sections is an important experimental challenge. Nevertheless, several experimental analyses have been collected for most simple case of MPI, i.e. the double parton scattering (DPS) $[1,2,3]$. From a theoretical point of view, we show that new fundamental information on the partonic proton structure $[4,5]$ could be accessed via DPS. Formally, the DPS cross section depends on the so called double parton distribution functions (dPDFs), $F_{i j}\left(x_{1}, x_{2}, \vec{z}_{\perp}, \mu\right)$, which describe the joint probability of finding two partons of flavors $i, j=q, \bar{q}, g$ with longitudinal momentum fractions $x_{1}, x_{2}$ and distance $\vec{z}_{\perp}$ in the transverse plane inside the hadron [6]. Here $\mu$ is the renormalization scale. Since no data are available for dPDFs, in order to estimate the magitudo of a given DPS process, unknown double parton correlations are usually neglected in experimental studies. However, the latter condition must be verified. In the present contribution we show how constituent quark model (CQM) calculations could be used to investigate the impact of double parton correlations (DPCs) in dPDFs (see Refs. $[5,7,8,9,10]$ ) and in experimental observables such as the so called $\sigma_{e f f}$. To this aim in Refs. $[8,11]$, dPDFs have been studied at the energy scale of the experiments and then used to calculate $\sigma_{e f f}[5,10,12,13,14]$. The DPS cross section, in processes with final state $A+B$, is written through the following ratio [15]:

$$
\sigma_{D P S}^{A+B}=\frac{m}{2} \frac{\sigma_{S P S}^{A} \sigma_{S P S}^{B}}{\sigma_{e f f}}
$$

where $m$ is combinatorial factor depending on the final states $A$ and $B$ ( $m=1$ for $A=B$ or $m=$ 2 for $A \neq B$ ) and $\sigma_{S P S}^{A(B)}$ is the single parton scattering cross section with final state $A(B)$. The present knowledge on DPS cross sections has been condensed in the experimental extraction of $\sigma_{e f f}[15,16,17,18,19,20,21,22,23,24,25]$. A constant value, $\sigma_{e f f} \simeq 15 \mathrm{mb}$, is compatible, within errors, with data: a result obtained neglecting DPCs. In the next sections the results of the calculations $\sigma_{e f f}$ within CQM will be described in order to characterize signals of DPCs.

\subsection{Double parton correlation and the same-sign $W W$ production at LHC}

In Refs. [5, 7, 8, 10], non perturbative correlations effects in dPDFs have been studied. We found out that DPCs cannot be easily neglected also in the experimental kinematic conditions. In Ref. [12], we have considered the same sign $W$ pair production process, a golden channel for the observation of DPS [26, 27], to establish to what extent double correlations could be accessed at the LHC. The differential DPS cross section can be written as follows [6]:

$$
d \sigma_{D P S}^{A B}=\frac{m}{2} \sum_{i, j, k, l} \int d \vec{z}_{\perp} F_{i j}\left(x_{1}, x_{2}, \vec{z}_{\perp}, \mu\right) F_{k l}\left(x_{3}, x_{4}, \vec{z}_{\perp}, \mu\right) d \hat{\sigma}_{i k}^{A} d \hat{\sigma}_{j l}^{B}
$$

where $\hat{\sigma}_{i j}^{A}$ represents the elementary cross section. As non perturbative input of the calculations, use has been made of dPDFs evaluated in Ref. [8]. Details of the fiducial DPS phase space adopted 
in the analysis and the theoretical errors are discussed in Ref. [12]. We found that the total $W$ charge summed DPS cross section (considering both $W$ decays into same sign muons) is found to be $\sigma^{++}+\sigma^{--}[\mathrm{fb}] \sim 0.69$. This result is consistent with those obtained by neglecting DPCs and those obtained with dPDFs of the model of Ref. [1]. The effects of DPCs have been investigated by observing the $\tilde{\sigma}_{e f f}$ dependence on $\eta_{1} \cdot \eta_{2} \simeq 1 / 4 \ln \left(x_{1} / x_{3}\right) \ln \left(x_{2} / x_{4}\right)$. For this process we found a mean value $\left\langle\widetilde{\sigma}_{e f f}\right\rangle \sim 21.04 \mathrm{mb}$, consistently with calculations of Refs. [5, 13, 14]. A clear signature of the presence of DPCs is found by observing the departure of $\tilde{\sigma}_{\text {eff }}$ from a constant, see right panel of Fig. 1. We have estimated that, with a luminosity of $\mathscr{L} \sim 1000 \mathrm{fb}^{-1}$, at $68 \%$ confidence level, the departure of $\sigma_{\text {eff }}$ from a constant value can be measured in the next run of the LHC.

\section{The $\sigma_{e f f}$ : a new window towards the transverse proton structure}

Here we show how the experimental estimates of $\sigma_{\text {eff }}$ could be interpreted in terms of the geometric structure of the proton. We consider the strategy used in Ref. [4], where DPCs have neglected in order to match with the experimental studies. In this scenario:

$$
\sigma_{e f f}^{-1}=\int d^{2} z_{\perp} T\left(z_{\perp}\right)^{2}=\int \frac{d^{2} k_{\perp}}{(2 \pi)^{2}} \tilde{T}\left(\vec{k}_{\perp}\right) \tilde{T}\left(-\vec{k}_{\perp}\right),
$$

where $\tilde{T}\left(k_{\perp}\right)$, called form factor (eff), is the Fourier transform (FT) of the probability of finding two partons with transverse distance $z_{\perp}$, i.e. $T\left(z_{\perp}\right)$. Thanks to the probabilistic interpretation of $T\left(z_{\perp}\right)$ and the asymptotic behaviour of $\tilde{T}\left(k_{\perp}\right), \sigma_{e f f}$ can be related to the mean transverse distance $\left\langle z_{\perp}^{2}\right\rangle$ between two partons in a DPS process [4, 5]. In fact $\left\langle z_{\perp}^{2}\right\rangle=-4 d \tilde{T}\left(k_{\perp}\right) /\left.d k_{\perp}^{2}\right|_{k_{\perp}=0}$. In the non relativistic limit:

$$
\tilde{T}\left(k_{\perp}\right)=\int d k_{1} d k_{1} \Psi^{\dagger}\left(\vec{k}_{1}+\vec{k}_{\perp}, \vec{k}_{2}\right) \Psi\left(\vec{k}_{1}, \vec{k}_{2}+\vec{k}_{\perp}\right),
$$

where $\vec{k}_{i}$ is the momentum of a parton $i$ and $\Psi$ is the proton wave function. The above expression is similar to the standard proton ff. However, in this case there is a double momentum imbalance, i.e. $k_{\perp}$. One should expect that in the extremely high $k_{\perp}$ region, the eff should fall to zero at least as the standard ff, being the eff a double ff $[4,5]$. Thanks to these general and model independent conditions, we found:

$$
\frac{\sigma_{e f f}}{3 \pi} \leq\left\langle z_{\perp}^{2}\right\rangle \leq \frac{\sigma_{e f f}}{\pi} .
$$

The above expression has been verified by using all models of dPDFs at our disposal, even for the pion target. In the right panel of Fig. 1 the experimental values of $\sigma_{e f f}$ have been used in Eq. (2.3) to get the mean transverse distance of the active partons. The above relation has been properly generalised in Ref. [5] in order to include correlations and splitting effects. Thanks to these analyses, new information on the transverse structure of the proton can be obtained from detailed experimental analyses of $\sigma_{\text {eff }}$.

\section{Conclusions}

In this contribution we have shown our main outcomes about dPDFs, remarking the impact of double parton correlations in experimental observables such as $\sigma_{\text {eff }}$. We found that the dependence 
of $\sigma_{\text {eff }}$ upon the longitudinal momentum fraction of the partons represents a clear sign correlations. We estimated that their effects could be observed in the next LHC run. From a different perspective, we have shown that new information on the mean transverse distance of partons inside the proton could be obtained from measurements of $\sigma_{e f f}$. All these studies suggest that dPDFs are fundamental tools to obtain new details on the non perturbative structure of the proton.
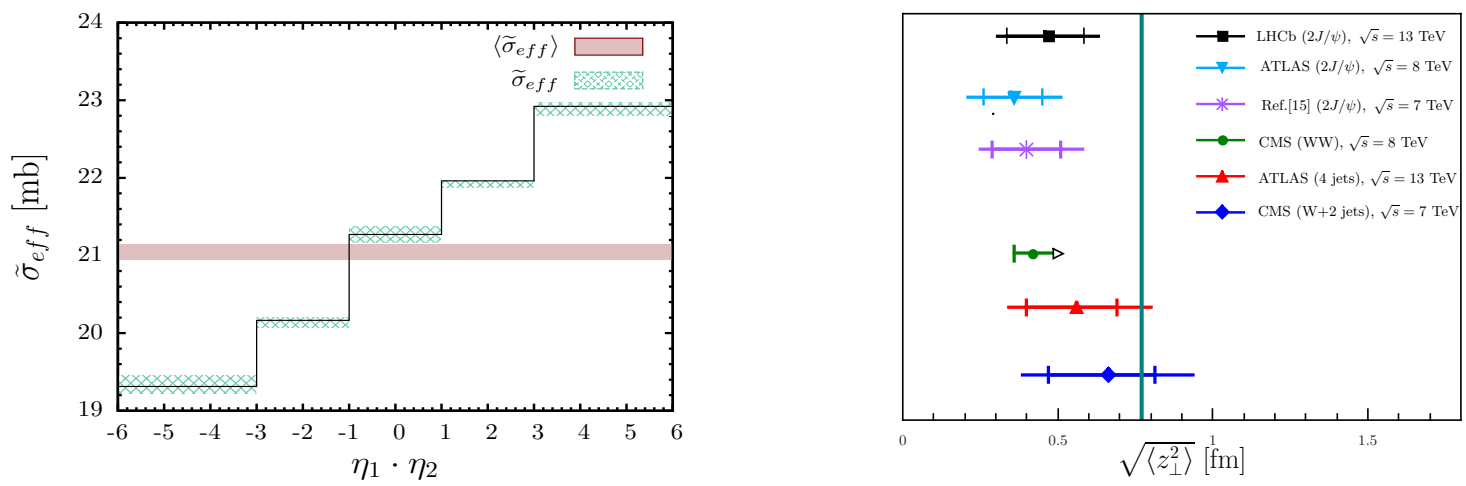

Figure 1: Left panel: $\tilde{\sigma}_{e f f}$ and $\left\langle\widetilde{\sigma}_{e f f}\right\rangle$ as a function of product of muon rapidities. Right panel: The application or Eq. (2.3) by using data of Refs. [20, 21, 22, 23, 24, 25].

\subsection{Acknowledgments}

The author thanks all the orginzers of the conference for the support given for this talk. The author also thanks F. A. Ceccopieri, S. Scopetta, M. Traini and V. Vento for their collaboration to this talk. This work was sup-ported in part by the STRONG-2020 project of the European Union's Horizon 2020 research and innovation programme under grant agreement No 824093.

\section{References}

[1] J. Gaunt and W. Stirling, Double Parton Distributions Incorporating Perturbative QCD Evolution and Momentum and Quark Number Sum Rules, JHEP 03 (2010) 005.

[2] M. Diehl, D. Ostermeier and A. Schafer, Elements of a theory for multiparton interactions in QCD, JHEP 03 (2012) 089 [1111.0910].

[3] P. Bartalini and L. Fano, eds., Proceedings, 1st International Workshop on Multiple Partonic Interactions at the LHC (MPIO8), (Hamburg), DESY, DESY, 2009. 10.3204/PUBDB-2017-128830.

[4] M. Rinaldi and F. A. Ceccopieri, Hadronic structure from double parton scattering, Phys. Rev. D97 (2018) 071501 [1801.04760].

[5] M. Rinaldi and F. A. Ceccopieri, Double parton scattering and the proton transverse structure at the LHC, JHEP 09 (2019) 097 [1812. 04286$].$

[6] N. Paver and D. Treleani, Multi - Quark Scattering and Large $p_{T}$ Jet Production in Hadronic Collisions, Nuovo Cim. 70 (1982) 215.

[7] M. Rinaldi, S. Scopetta and V. Vento, Double parton correlations in constituent quark models, Phys. Rev. 87 (2013) 114021 [1302.6462]. 
[8] M. Rinaldi, S. Scopetta, M. Traini and V. Vento, Double parton correlations and constituent quark models: a Light Front approach to the valence sector, JHEP 12 (2014) 028 [1409.150 0].

[9] A. Courtoy, S. Noguera and S. Scopetta, Double parton distributions in the pion in the Nambu-Jona-Lasinio model, JHEP 12 (2019) 045 [1909. 09530].

[10] M. Rinaldi and F. A. Ceccopieri, Relativistic effects in model calculations of double parton distribution function, Phys. Rev. 95 (2017) 034040 [1611 . 04793 ].

[11] M. Rinaldi, S. Scopetta, M. C. Traini and V. Vento, Correlations in Double Parton Distributions: Perturbative and Non-Perturbative effects, JHEP 10 (2016) 063 [1608. 02521].

[12] F. A. Ceccopieri, M. Rinaldi and S. Scopetta, Parton correlations in same-sign W pair production via double parton scattering at the LHC, Phys. Rev. 95 (2017) 114030 [1702 . 05363].

[13] M. Rinaldi, S. Scopetta, M. Traini and V. Vento, Double parton scattering: a study of the effective cross section within a Light-Front quark model, Phys. Lett. 752 (2016) 40 [1506 . 05742].

[14] M. Traini, M. Rinaldi, S. Scopetta and V. Vento, The effective cross section for double parton scattering within a holographic AdS/QCD approach, Phys. Lett. 768 (2017) 270 [1609 . 07242 ].

[15] H. Jung, D. Treleani, M. Strikman and N. van Buuren, eds., Proceedings, 7th International Workshop on Multiple Partonic Interactions at the LHC (MPI@LHC 2015), 2016.

[16] Axial FIEld Spectrometer collaboration, Double Parton Scattering in pp Collisions at $\sqrt{s}=63-G e V, Z$. Phys. 34 (1987) 163.

[17] UA2 collaboration, A Study of multi - jet events at the CERN anti-p p collider and a search for double parton scattering, Phys. Lett. 268 (1991) 145.

[18] CDF collaboration, Double parton scattering in $\bar{p} p$ collisions at $\sqrt{s}=1.8 T e V$, Phys. Rev. 56 (1997) 3811.

[19] D0 collaboration, Double parton interactions in $\gamma+3$ jet events in $p p^{-}$bar collisions $\sqrt{s}=1.96 \mathrm{TeV}$., Phys. Rev. 81 (2010) 052012 [0912.5104].

[20] LHCB collaboration, Measurement of the J/ $\psi$ pair production cross-section in pp collisions at $\sqrt{s}=13 \mathrm{TeV}, \mathrm{JHEP} 06(2017) 047$ [1612.07451].

[21] CMS collaboration, Study of double parton scattering using $W+2$-jet events in proton-proton collisions at $\sqrt{s}=7 \mathrm{TeV}$, JHEP $\mathbf{0 3}$ (2014) 032 [1312 . 5729].

[22] ATLAS collaboration, Study of hard double-parton scattering in four-jet events in pp collisions at $\sqrt{s}=7 \mathrm{TeV}$ with the ATLAS experiment, JHEP 11 (2016) 110 [1608.01857].

[23] CMS collaboration, Constraints on the double-parton scattering cross section from same-sign $W$ boson pair production in proton-proton collisions at $\sqrt{s}=8 \mathrm{TeV}, \mathrm{JHEP} 02$ (2018) 032 [1712.02280].

[24] ATLAS collaboration, Measurement of the prompt J/ $\psi$ pair production cross-section in pp collisions at $\sqrt{s}=8 \mathrm{TeV}$ with the ATLAS detector, Eur. Phys. J. 77 (2017) 76 [1612 . 02950].

[25] J.-P. Lansberg and H.-S. Shao, J/ $\psi$-pair production at large momenta: Indications for double parton scatterings and large $\alpha_{s}^{5}$ contributions, Phys. Lett. 751 (2015) 479 [1410 . 8822].

[26] J. R. Gaunt, C.-H. Kom, A. Kulesza and W. J. Stirling, Same-sign W pair production as a probe of double parton scattering at the LHC, Eur. Phys. J. 69 (2010) 53 [1003. 3953].

[27] S. Cotogno, T. Kasemets and M. Myska, Spin on same-sign W -boson pair production, Phys. Rev. 100 (2019) 011503 [1809.09024]. 